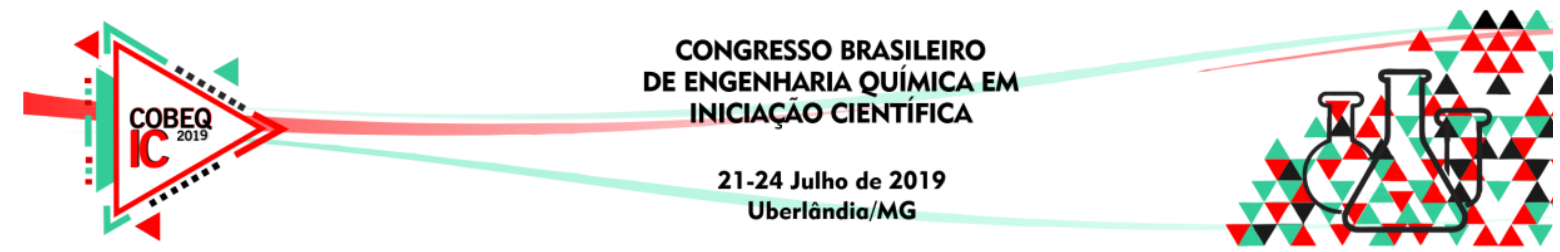

\title{
DESENVOLVIMENTO DE UM MODULO DIDÁTICO DE PERDA DE CARGA PARA ENSINO DE MECÂNICA DOS FLUIDOS EM ENGENHARIA
}

\author{
S. L. M. R. CARNEIRO ${ }^{1}$, A. ESTEVES ${ }^{1}$, E. G. L. DE CARVALHO ${ }^{1}$ e A. B. DOS REIS ${ }^{1}$ \\ ${ }^{2}$ Universidade Federal dos Vales do Jequitinhonha e Mucuri, Instituto de Engenharia \\ egstephane@gmail.com
}

\begin{abstract}
RESUMO - Estudar a perda de carga é de fundamental importância para o correto dimensionamento de sistemas de bombeamento e tubulações. Assim, o principal objetivo é a construção de um modulo didático de perda de carga com aquisição de dados para criação de experimentos e roteiros a serem usados em planta laboratorial, incluindo estudos que contemplam avaliar a eficiência do módulo, cálculos de altura de projeto, a pressão absoluta exercida pelo sistema na sucção e elaboração da curva característica da bomba.
\end{abstract}

\section{INTRODUÇÃO}

O módulo didático representa um protótipo de um sistema que realiza o transporte de um fluido entre reservatórios de diferentes alturas, semelhante ao processo de abastecimento de água proveniente de poços artesianos e de estações de tratamento de água e efluentes (Souza, 2014). O bombeamento pode ser de água ou de qualquer outro fluido, mas geralmente as especificações do fabricante são referentes a água como fluido de trabalho. Vale destacar, que fluidos como o enxofre, em que a viscosidade é superior à da água, os parâmetros como ponto ótimo de operação da bomba devem ser corrigidos. Sendo que, fluidos mais viscosos podem acarretar uma redução da eficiência da bomba e aumento da potência absorvida, além da redução da altura manométrica e redução da vazão (Gomes, 2013).

Para realizar o correto dimensionamento de sistemas de bombeamento é necessário dominar cálculos, como por exemplo, de perda de carga. Além disso, determinar qual a bomba adequada para a finalidade do sistema, envolvendo o conhecimento do ponto ótimo de operação, obtido por meio da curva característica da bomba. Visto que, trabalhar no ponto ótimo indica maior eficiência e menores custos de operação. Já o (NPSHD) equivale a pressão absoluta exercida pelo sistema na sucção e indica se ocorrerá o fenômeno de cavitação na bomba quando comparado ao $\left(\mathrm{NPSH}_{\mathrm{R}}\right)$ pressão mínima exigida pela bomba na sucção (Cremasco, 2012).

Desse modo, o presente trabalho tem como objetivo desenvolver um módulo didático de perda de carga que irá auxiliar as aulas de laboratório de engenharia estudando cálculos de perda de carga localizada e distribuída, pressão absoluta requerida na entrada da bomba $\left(\mathrm{NPSH}_{\mathrm{D}}\right)$, potência e eficiência da bomba, assim como a construção da curva característica da bomba. Com isso, é possível explorar parâmetros importantes tanto no ramo industrial quanto acadêmico. 


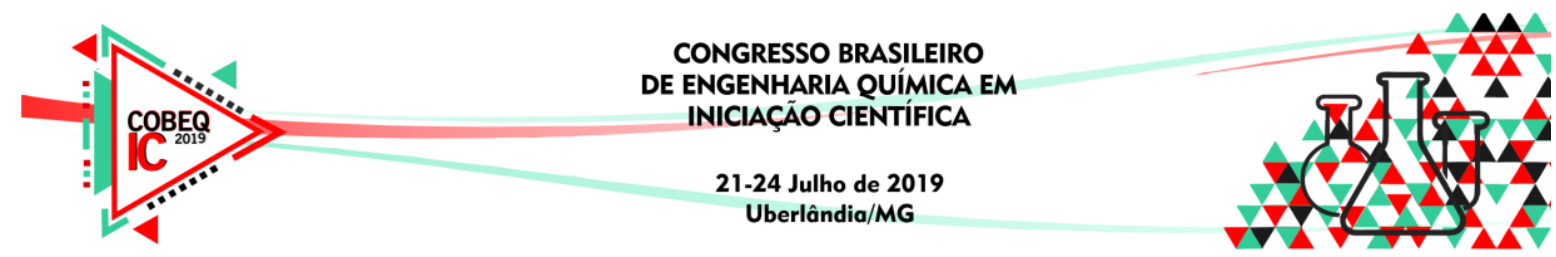

\section{METODOLOGIA}

\subsection{O Experimento}

O módulo didático desenvolvido é composto por quatro tês, três joelhos, oito conexões, uma válvula gaveta, uma válvula de retenção e uma saída de canalização, incluindo um comprimento reto de aproximadamente $223,5 \mathrm{~cm}$. Sendo todos esses elementos necessários para conduzir estudos extensos de perdas dianteiras em tubos e válvulas. O módulo é ilustrado através da Figura 1.

Figura 1 - Esquema do Módulo Didático.

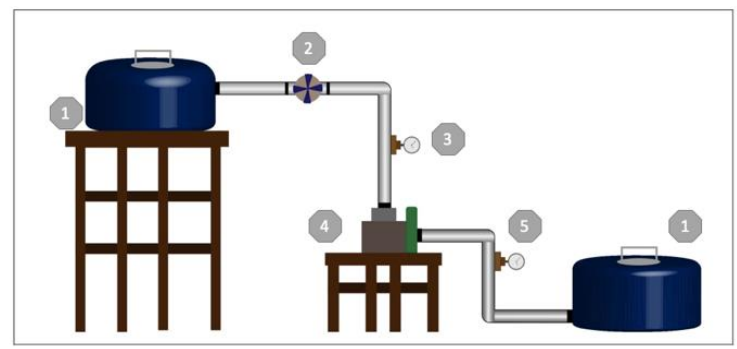

Inicialmente foi realizado um levantamento bibliográfico sobre módulos hidráulicos de perda de carga buscando compreender as especificações, relação de acessórios e tubulações de acordo com a norma técnica para policloreto de polivinila (PVC).

Alguns ensaios foram elaborados para determinação de parâmetros importantes, como, tempo, área do reservatório e abertura de válvulas. Realizou-se seis ensaios em triplicata no módulo didático, aumentando a vazão de escoamento no decorrer do experimento conforme a abertura da válvula gaveta em $1 / 4 ; 1 / 2 ; 3 / 4 ; 1 ; 1$ e 1/4; 1 e $1 / 2$ de volta em um tempo de 10 segundos para cada abertura. Com a determinação desses parâmetros obteve-se os dados para calcular a perda de carga localizada em cada um dos respectivos acessórios e a perda de carga distribuída em todo o módulo. Assim foi possível detectar quais acessórios mais contribuem para a perda de carga. Ademais, com os resultados obtidos foi possível validar a eficiência do módulo construído, através da determinação do $\mathrm{NPSH}_{D}$, potência e construção da curva característica da bomba.

\subsection{Parâmetros Analisados}

Na perda de carga distribuída as paredes dos dutos retilíneos causam uma perda de pressão ao longo do tubo, fazendo com que a pressão total vá diminuindo gradativamente ao longo de seu comprimento. Essa perda de carga depende do diâmetro, do comprimento do tubo, da rugosidade da parede, das propriedades do fluido, da massa específica, da viscosidade e da velocidade de escoamento do fluido. A perda de carga distribuída pode ser calculada conforme a Equação 1. 


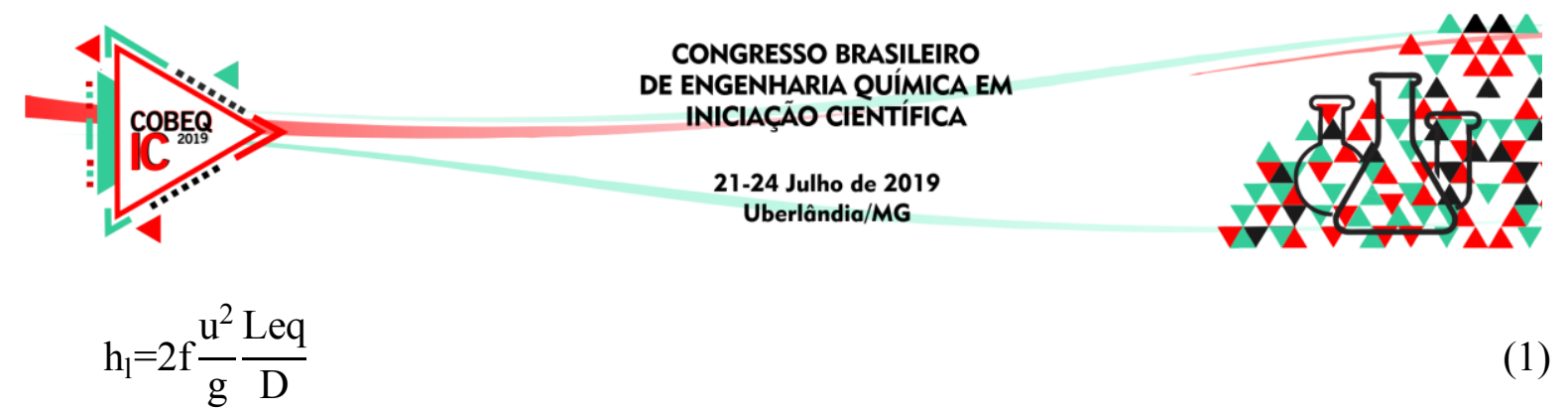

Onde, o Leq é definido como um comprimento equivalente da tubulação que causa a mesma perda de carga que o acessório, esse foi calculado conforme (Cremasco, 2012). A rugosidade possui o valor de 0,0000015 m conforme NBR 5626. O fator de fricção f é expresso em função da rugosidade, diâmetro da tubulação e regime de escoamento, sendo que, em sistemas turbulentos esse fator pode ser determinado de acordo com a Equação 2.

$$
\mathrm{f}=\left\{\left(\frac{64}{\mathrm{Re}}\right)^{8}+9,5 \cdot\left[\ln \left(\frac{\varepsilon}{3,7 \cdot \mathrm{D}}+\left(\frac{2500}{\mathrm{Re}^{0,9}}\right)^{6}\right)\right]^{-16}\right\}^{0,125}
$$

A perda de carga localizada é causada pelos acessórios de canalização. Experimentalmente observa-se que a perda de carga em acessórios é constante quando o regime é turbulento, podendo ser determinada pela Equação 3.

$$
\mathrm{h}_{\mathrm{p}}=\mathrm{k} \frac{\mathrm{u}^{2}}{2 \mathrm{~g}}
$$

Para especificar uma bomba para uma dada aplicação é de fundamental importância determinar a altura de projeto, esse parâmetro pode ser calculado conforme (Cremasco, 2012). Além disso também é de suma importância determinar a potência da bomba, pois está associada ao gasto de energia necessário para deslocar o fluido. A relação entre potência útil de uma bomba e a potência consumida fornece o rendimento, obtido conforme a Equação 4.

$$
\text { Rendimento }=\frac{\mathrm{W}_{\mathrm{u}}}{\mathrm{W}_{\text {consumido }}}
$$

Nas operações de bombeamento a altura de sucção disponível $\left(\mathrm{NPSH}_{\mathrm{D}}\right)$ indica a condição ideal para que ocorra a sucção do fluido. Uma vez que, existe um limite de pressão de vácuo que pode ser atingido na sucção de uma bomba, de modo, que abaixo desse limite ocorrerá o fenômeno de cavitação (Brunetti, 2008). O NPSHDé calculado conforme a Equação 5.

$$
\mathrm{NPSH}_{\mathrm{D}}=\frac{\text { PA-Pvapor }}{\rho \mathrm{g}}+\mathrm{H}_{\mathrm{s}}
$$

Esse parâmetro e fornecido pelo fabricante em função da vazão e quando o NPSH disponível for menor igual ao requerido a cavitação ocorrerá (Cremasco, 2012).

Além dos parâmetros supracitados, tem-se também as curvas características da bomba, que tem como objetivo descrever as condições operacionais, especificamente de bombas centrífugas. Essas curvas são diagramas que usualmente contém a altura de projeto e a vazão de trabalho. 


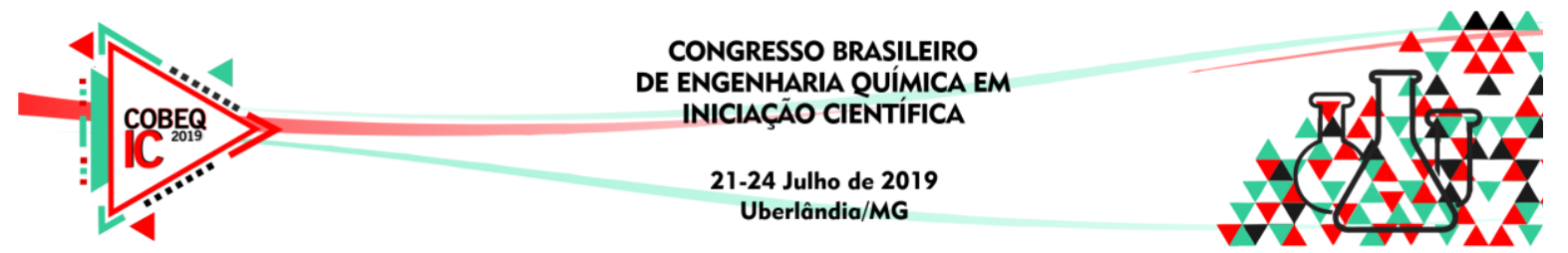

\section{RESULTADOS}

Na Tabela 1 encontra-se os valores referentes à perda de carga distribuída encontrada através da Equação 1, tendo em vista que o diâmetro da tubulação é de $13 \frac{3}{4}$ in.

Tabela 1-Perda de Carga Distribuída

\begin{tabular}{|c|c|}
\hline Vazão $\left(\mathbf{m}^{\mathbf{3}} / \mathbf{s}\right)$ & $\mathbf{h l} \mathbf{( m )}$ \\
\hline 0,000058 & 0,005030 \\
\hline 0,000267 & 0,020280 \\
\hline 0,000592 & 0,105020 \\
\hline 0,000857 & 0,224890 \\
\hline 0,001010 & 0,312820 \\
\hline 0,001220 & 0,464000 \\
\hline
\end{tabular}

Observa-se que a perda de carga aumenta proporcionalmente com a vazão, sendo que a determinação do fator de fricção depende do número de Reynolds, logo quanto maior a vazão com maior turbulência o fluido passa pela tubulação.

Já a Tabela 2 demonstra a perda de carga localizada nos assessórios com diâmetro de 3/4 e 1 in.

Tabela 2 - Perda de Carga Localizada

\begin{tabular}{|c|c|c|}
\hline Vazão $\left(\mathbf{m}^{\mathbf{3}} / \mathbf{s} \mathbf{)}\right.$ & hf $\mathbf{3} / \mathbf{~ i n}$ & hf $\mathbf{1}$ in \\
\hline 0,000058 & 0,0634 & 0,0192 \\
\hline 0,000267 & 1,5156 & 0,4575 \\
\hline 0,000592 & 7,8920 & 2,3794 \\
\hline 0,000857 & 16,9525 & 5,1085 \\
\hline 0,001010 & 23,6134 & 7,1141 \\
\hline 0,001220 & 35,0872 & 10,5681 \\
\hline
\end{tabular}

Nota-se que quanto menor o diâmetro maior é o valor de perda de carga associada. Isto porque o fluido terá que realizar contração e expansão ao passar pelos assessórios, visto que há uma discrepância entre o diâmetro da tubulação e do acessório, estes fenômenos são responsáveis por turbilhões que causam dissipação da energia potencial e consequentemente acarretam uma perda carga associada (Fox et al., 2010).

A Tabela 3 apresenta os valores encontrados para potência e rendimento da bomba nas diferentes vazões trabalhadas. Considerando o $\mathrm{W}$ consumido como $370 \mathrm{Watts}$, sendo este valor fornecido pelo fabricante. 


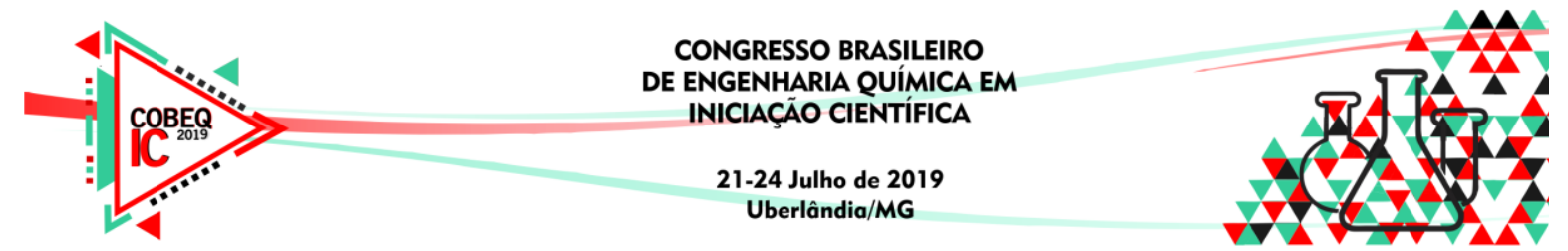

Tabela 3 - Potência Útil e Rendimento da Bomba em Função da Vazão

\begin{tabular}{|c|c|c|}
\hline Vazão $\left(\mathbf{m}^{\mathbf{3}} \mathbf{s}\right)$ & Potência (Watts) & Rendimento \\
\hline 0,000058 & 9,57 & $2,59 \%$ \\
\hline 0,000267 & 40,50 & $10,95 \%$ \\
\hline 0,000592 & 84,02 & $22,71 \%$ \\
\hline 0,000857 & 106,78 & $28,86 \%$ \\
\hline 0,001010 & 111,17 & $30,05 \%$ \\
\hline 0,001220 & 108,24 & $29,25 \%$ \\
\hline
\end{tabular}

Com os resultados obtidos nota-se um rendimento baixo da bomba, o que pode ser justificado devido a bomba ter operado numa potência inferior à sua capacidade. Os reservatórios presentes no módulo didático possuem um volume reduzido, o que limita a utilização da bomba em vazões superiores no tempo de $10 \mathrm{~s}$.

Na Tabela 4 tem-se os valores de altura de sução, descarga manométrica e $\mathrm{NPSH}_{\mathrm{D}}$ em função das vazões de trabalho.

Tabela 4 - Altura Manométrica e NPSHD em Função da Vazão

\begin{tabular}{|c|c|c|c|c|}
\hline H sucção (m) & H descarga (m) & H manométrica $(\mathbf{m})$ & Vazão $\left(\mathbf{m}^{3} / \mathbf{s}\right)$ & NPSHo $(\mathbf{m})$ \\
\hline 0,014693 & 16,895820 & 16,881126 & 0,000058 & 10,027448 \\
\hline 0,060289 & 15,523648 & 15,463359 & 0,000267 & 10,073044 \\
\hline 0,213036 & 14,687652 & 14,474616 & 0,000592 & 10,225791 \\
\hline 0,735027 & 13,452434 & 12,717406 & 0,000857 & 10,747782 \\
\hline 1,149379 & 12,430112 & 11,280733 & 0,001010 & 11,162134 \\
\hline 2,082798 & 11,152872 & 9,070074 & 0,001220 & 12,095553 \\
\hline
\end{tabular}

Segundo a literatura, a altura manométrica do sistema corresponde a energia que o sistema exige da bomba para transferir um fluido de um ponto a outro a uma certa vazão. Porém essa energia pode variar levando-se em conta as resistências que este sistema fornece ao fluido. Tais resistências são: a diferença entre os níveis dos reservatórios de descarga e sucção, diferença de pressão entre os reservatórios de descarga e sucção e as perdas de carga do sistema. Além disso, o cálculo da altura manométrica total é feito considerando-se o quanto de energia já existe na linha de sucção e o quanto de energia se deve ter na linha de descarga (Souza, 2014).

Da mesma forma que a perda de carga o $\mathrm{NPSH}_{\mathrm{D}}$ aumenta proporcionalmente com a vazão, o que indica que quanto maior a vazão mais próxima a bomba estará de cavitar. $\mathrm{Na}$ realização do experimento não foi observado o fenômeno, que é caracterizado por um som característico advindo da bomba. Afirmar com certeza a respeito da cavitação não foi possível, pois o $\mathrm{NPSH}_{\mathrm{R}}$ é um dado fornecido pelo fabricante, dado este que não foi possível obter.

Por fim a curva característica da bomba é apresentada no Gráfico 1. Através dele é possível observar que a curva dada pelo fabricante e a experimental apresentaram comportamento semelhante. 


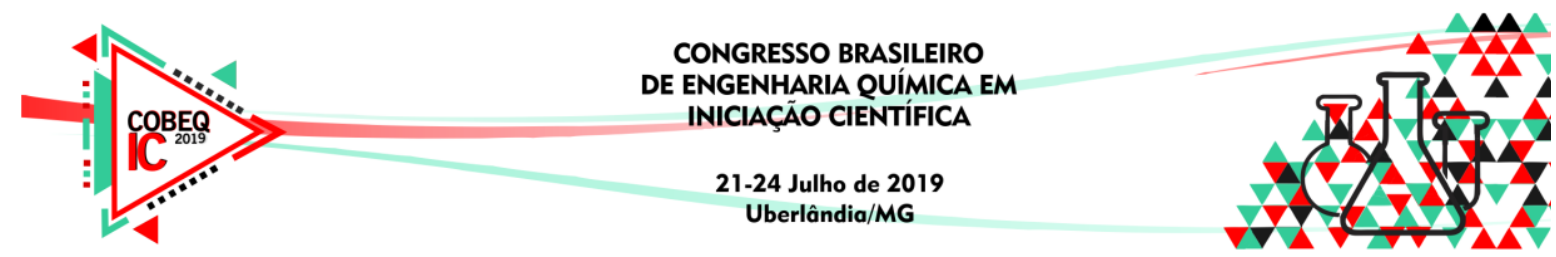

Gráfico 1 - Curva da Bomba (Q x H)

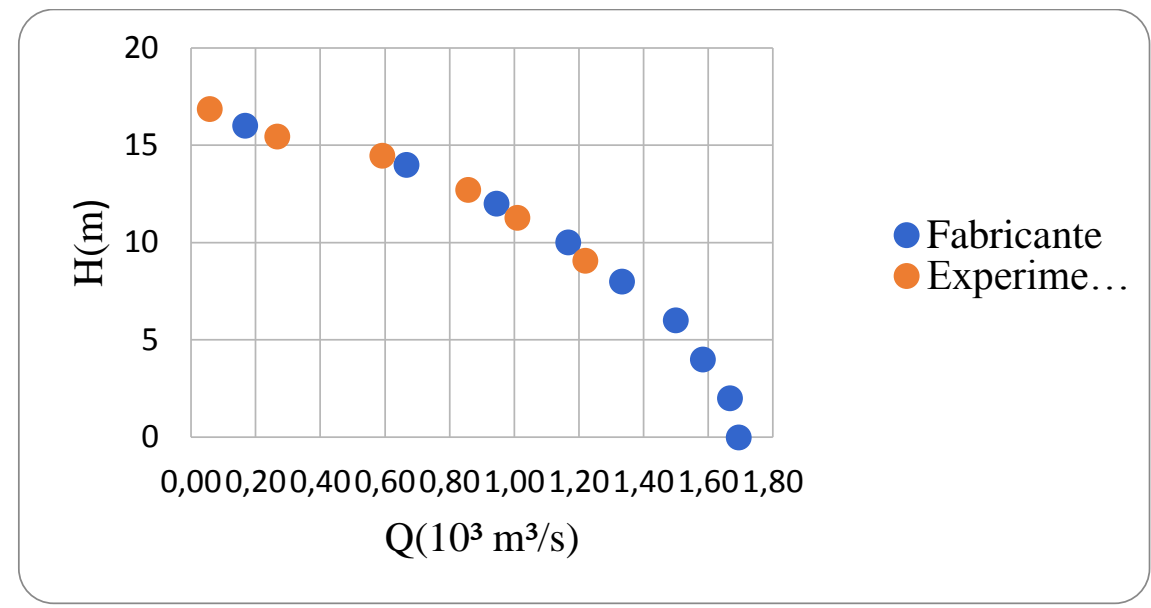

\section{CONCLUSÃO}

Com o desenvolvimento do módulo didático foi possível estudar a perda de carga localizada e distribuída, $\mathrm{NPSH}_{\mathrm{D}}$, potência e rendimento da bomba, bem como a construção da curva característica da bomba.

\section{REFERÊNCIAS}

ASSOCIAÇÃO BRASILEIRA DE NORMAS TÉCNICAS. NBR 5626: Instalação predial de água fria. Rio de Janeiro, 1998.

BRUNETTI, F., Mecânica dos Fluidos. São Paulo: Editora Eugênia Pessotti, 2008.

CREMASCO, M.A. Operações Unitárias em Sistemas Particulados e Fluido Mecânicos. São Paulo, Editora Blucher, 2012.

SOUZA, P, H, A. I. Apresentação dos cálculos para seleção de bomba para sistema de reaproveitamento de água de poços artesianos. Universidade Federal do Rio de JaneiroDepartamento de Engenharia Mecânica. Rio de Janeiro, 2014.

FOX, R. W.; MCDONALD, A. T.; PRITCHARD, P. J. Introdução a Mecânica dos fluidos. Rio de Janeiro, LTC, 2010.

GOMES, G. A. P. M. Seleção de Bomba Centrífuga Vertical para Operação em um Sistema de Transferência de Enxofre Líquido. Projeto de Graduação, Rio de Janeiro: UFRJ/Escola Politécnica, 2013 